\title{
Correction to: Uniformly Compressing Mean Curvature Flow
}

\section{Wenhui Shi ${ }^{1} \cdot$ Dmitry Vorotnikov ${ }^{1}$}

Published online: 29 January 2022

(c) Mathematica Josephina, Inc. 2022

\section{Correction to: The Journal of Geometric Analysis (2019) 29:3055-3097 https://doi.org/10.1007/s12220-018-00104-z}

In the sketch of the proof of Theorem 4 we claimed that a weak solution $(\xi, \kappa, \sigma)$ can be obtained by following the same lines as in the proof of [1, Proposition 3.4 and Theorem 3]. However, an appropriate argument should be slightly different due to different boundary conditions. The aim of this addendum is to fill that gap.

More precisely, in [1] at the free end one has $\sigma^{\epsilon}(t, 0)=\kappa^{\epsilon}(t, 0)=0$ for all $t \in[0, T]$. Therefore, the uniform (w.r.t. to $\epsilon) L^{2}$ bound for $\left(\sigma^{\epsilon}, \kappa^{\epsilon}\right)$ follows directly from the Poincaré inequality and the uniform $L^{2}$ bound for $\left(\partial_{s} \sigma^{\epsilon}, \partial_{s} \kappa^{\epsilon}\right)$. In our case, the vanishing boundary conditions for $\sigma^{\epsilon}$ and $\kappa^{\epsilon}$ are no longer satisfied. We thus need some additional estimates to obtain the uniform $L^{2}$ bound.

We first estimate the spatial average $\overline{\sigma^{\epsilon}}(t):=\int_{\mathbb{S}^{1}} \sigma^{\epsilon}(s, t) \mathrm{d} s$. Indeed, an integration by parts and Cauchy-Schwarz yield

$$
\left|\overline{\sigma^{\epsilon}}(t)\right|=\left|\int_{\mathbb{S}^{1}} \kappa^{\epsilon} \cdot \partial_{s} \xi^{\epsilon} \mathrm{d} s\right|=\left|-\int_{\mathbb{S}^{1}} \partial_{s} \kappa^{\epsilon} \cdot \xi^{\epsilon} \mathrm{d} s\right| \leq\left\|\partial_{S} \kappa^{\epsilon}(t, \cdot)\right\|_{L^{2}\left(\mathbb{S}^{1}\right)}\left\|\xi^{\epsilon}\right\|_{L^{2}\left(\mathbb{S}^{1}\right)}, \forall t \in[0, T]
$$

Thus

$$
\left\|\overline{\sigma^{\epsilon}}\right\|_{L^{2}([0, T])} \leq \sup _{t \in[0, T]}\left\|\xi^{\epsilon}(t, \cdot)\right\|_{L^{2}\left(\mathbb{S}^{1}\right)}\left\|\partial_{S} \kappa^{\epsilon}\right\|_{L^{2}\left(Q_{T}\right)}
$$

where the right-hand side is uniformly bounded (cf. [1, Proposition 3.1]). Thus from Poincaré inequality $\left\|\sigma^{\epsilon}-\overline{\sigma^{\epsilon}}\right\|_{L^{2}\left(Q_{T}\right)} \leq C(T)\left\|\partial_{S} \sigma^{\epsilon}\right\|_{L^{2}\left(Q_{T}\right)}$ we obtain $\left\|\sigma^{\epsilon}\right\|_{L^{2}\left(Q_{T}\right)} \leq$ $C$ with $C$ independent of $\epsilon$.

The original article can be found online at https://doi.org/10.1007/s12220-018-00104-z.

$凶$ Dmitry Vorotnikov

mitvorot@mat.uc.pt

Wenhui Shi

wshi@mat.uc.pt

1 CMUC, Department of Mathematics, University of Coimbra, 3001-501 Coimbra, Portugal 
It remains to show that $\kappa^{\epsilon}$ is uniformly bounded in $L^{2}\left(Q_{T}\right)$. To this end, we note that from the definition of $\kappa^{\epsilon}$ one has $\partial_{s} \xi^{\epsilon}=\epsilon \kappa^{\epsilon}+\frac{\kappa^{\epsilon}}{\sqrt{\epsilon+\left|\kappa^{\epsilon}\right|^{2}}}$, whence

$$
\sigma^{\epsilon}=\kappa^{\epsilon} \cdot \partial_{s} \xi^{\epsilon}=\left|\kappa^{\epsilon}\right|\left(\epsilon\left|\kappa^{\epsilon}\right|+\frac{\left|\kappa^{\epsilon}\right|}{\sqrt{\epsilon+\left|\kappa^{\epsilon}\right|^{2}}}\right) \geq\left|\kappa^{\epsilon}\right|\left|\partial_{s} \xi^{\epsilon}\right|
$$

Observing that

$$
\left|\partial_{s} \xi^{\epsilon}\right|=\epsilon\left|\kappa^{\epsilon}\right|+\frac{\left|\kappa^{\epsilon}\right|}{\sqrt{\epsilon+\left|\kappa^{\epsilon}\right|^{2}}} \geq \epsilon+\frac{1}{\sqrt{1+\epsilon}}>1
$$

provided $\left|\kappa^{\epsilon}\right| \geq 1$, we infer that $\left|\kappa^{\epsilon}\right| \leq \sigma^{\epsilon}$ when $\left|\kappa^{\epsilon}\right| \geq 1$. Thus,

$$
\begin{aligned}
\int_{Q_{T}}\left|\kappa^{\epsilon}\right|^{2} \mathrm{~d} s \mathrm{~d} t & =\int_{\left\{(s, t) \in Q_{T}:\left|\kappa^{\epsilon}\right|<1\right\}}\left|\kappa^{\epsilon}\right|^{2} \mathrm{~d} s \mathrm{~d} t+\int_{\left\{(s, t) \in Q_{T}:\left|\kappa^{\epsilon}\right| \geq 1\right\}}\left|\kappa^{\epsilon}\right|^{2} \mathrm{~d} s \mathrm{~d} t \\
& \leq\left|Q_{T}\right|+\int_{Q_{T}}\left|\sigma^{\epsilon}\right|^{2} \mathrm{~d} s \mathrm{~d} t \leq C,
\end{aligned}
$$

where $C$ does not depend on $\epsilon$.

\section{Reference}

1. Shi, W., Vorotnikov, D.: The gradient flow of the potential energy on the space of arcs. Calc. Var. 58, 59 (2019)

Publisher's Note Springer Nature remains neutral with regard to jurisdictional claims in published maps and institutional affiliations. 